\title{
Dry Coating Method Using Magnetically Assisted Impaction In a Randomly Turbulent Fluidized Bed ${ }^{+}$
}

\author{
Rajiv K. Singh, A. Ata, and J. Fitz-Gerald \\ and Ya.I. Rabinovich \\ Department of Materials Science and Engineering \\ University of Florida* \\ W. Hendrickson \\ Aveka Inc**
}

\begin{abstract}
Surface modified particulates have many potential industrial applications ranging from new technologies such as rechargeable batteries, flat-panel displays, etc. to a wide range of unit operation processes such as dispersion, transport and handling, and separation of particulate systems. Due to environmental constraints, there has been a strong interest in the development of dry methods (chemical and/or water free) for particulate coatings. In this paper, we report the feasibility of novel dry method based on a magnetically-assisted impaction coating (MAIC) process for synthesis of composite particulates. In the MAIC process, magnetic particles are accelerated in the chamber using an alternating electromagnetic field. The magnetic particles in turn collide with the core and with submicron sized fine particles (secondary particles) to form composite particles. The adhesion of the secondary particles on the core particles was found to be dependent on several factors including particle size, particle hardness, etc. and a number of processing parameters. Experiments were conducted on a wide variety of particles systems such $\mathrm{TiO}_{2} / \mathrm{PMMA}$, Alumina/PMMA, Ag/PMMA, TiO $\mathrm{O}_{2} / \mathrm{Al}_{2} \mathrm{O}_{3}$, etc to understand the effect of these parameters. PMMA were preferred to use as a core (primary) particle because of its smooth surface which minimizes surface roughness effects. The composite particles were characterized using standard materials techniques such as scanning electron microscopy, energy dispersive $X$-ray microanalysis, etc. The results show that MAIC process significantly improved the surface coverage compared to the coverage obtained from standard blending methods. High frequency ultra-sonication of composite particles was also conducted so that weakly adherent particles could be removed from the surface. The efficiency of the coating process was found to be decreased with increasing secondary particle size. High surface coverage was achieved for composite particle with soft cores (e.g PMMA). To understand the effect of particle hardness on the adhesion process, atomic force microscopy (AFM) studies were conducted as a function of the particle hardness. Based on experimental observations, a model for the particle coating process has been developed.
\end{abstract}

\section{Introduction}

The synthesis of engineered particulates i.e. par ticulates with tailored properties, have become increasingly important for a variety of industrial and technological applications (1-2). Methodologies are being developed to synthesize particulates with desired properties such as specific size, size distribution, and shape, etc. One important class of engineered particulates are composite particles in

* Gainesville, FL 32611, USA

**2045 Wooddale Drive, Woodbury, MN 55125

+ Received October, 1996 which the surface properties of the particle can be tailored for various applications. Fig. 1 is a schematic diagram of the two types of engineered particulates with controlled surface architecture. The secondary particle adheres to the surface of the core particle, thereby modifying its surface properties. Various inter-particle forces such as Van der Waals, electrostatic, capillary forces, etc, are important in controlling the adhesion of the secondary particle to the core particle. Two different types of surface architecture are depicted in this diagram. In the first case, the secondary particles 


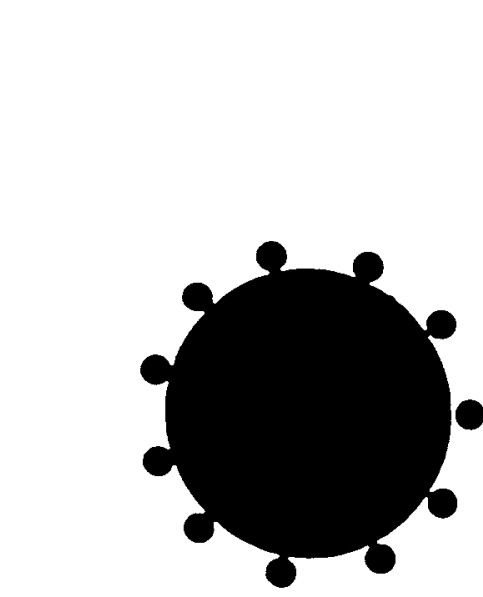

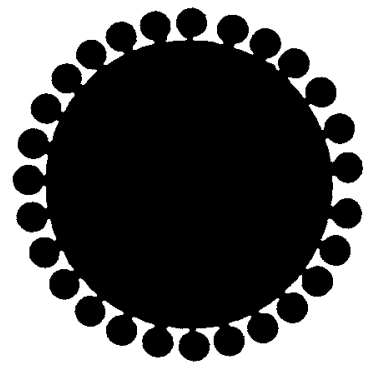

Fig. 1 Schematic diagram of (i) discrete and (ii) continuous engineered particulates with controlled surface architecture

form a continuous coating on the surface, while in the second case, a discrete discontinuous coating is formed. The size of the secondary particle can vary from $\sim 1 \mathrm{~nm}$ to $>1 \mu \mathrm{m}$, while the size of the core particle can range from less than $1 \mu \mathrm{m}$ to greater than $200 \mu \mathrm{m}$. Both discrete and continuous coatings may be important, depending on the nature of the application.

This class of engineered particulate has wideranging applications extending from synthesis of ceramic tungsten carbide/cobalt cutting tools, metal hydride based rechargeable batteries, to phosphors for flat panel display materials. In each of these application, it is desirable to obtain a surface property which is different from the bulk particle. To achieve this, particulate surfaces have to be designed so that the surface properties (e.g conductivity, sinterability, corrosion characteristics, flow behavior, etc.) are different from the bulk material. For example, the surface of phosphor powders should be conducting so that the charge introduced by cathodoluminescence can be dissipated, while in $\mathrm{WC} / \mathrm{Co}$ cutting tools, the surface has to modified so that high-density sintering of the ceramic can be achieved at relatively lower processing temperatures. Also, the surfaces can be designed to have novel properties such as selective adsorption, high catalytic activity, etc.. Thus, the synthesis of specifically engineered particulates is expected to play an important role in the next generation particulate technologies. It should also be noted that by controlling the surface architecture of the coating, segregation effects can be also minimized. A uniformly coated composite particle represents a structured mixture of two systems (3-5).

Synthesis of engineered particulates of controlled architecture can be accomplished by both wet and dry processes. Typically in wet processes, the core particles are dispersed in an aqueous/non-aqueous

solution, before the coating with the secondary particle takes place. The secondary particles can exist as second suspended phase before the mixing process, or can be in-situ precipitated on to the core particles. Wet techniques are characterized by the use of chemicals/water for the process. These techniques rely on the use of electrostatic, Van der Waals and steric forces for adherence of the secondary particle on the core particle.

Recently, there has been a concerted effort to develop coating processes which do not require the use of chemicals, or aqueous/non aqueous solvents. The development of new and existing "dry processes" for particulate coatings has become crucial for the development of next generation particulate coating technologies. Typically dry methods to synthesize composite particles use some type of grinding system. Almost every fine grinding machine appears to possess the ability to make various kinds of composite particles, aside from its primary function. To make the composite particles, the secondary particle is initially covered over the surface of the core particle, and then mechanical compressive and shear stresses are applied to enhance the adhesion process. There are a number of reports which claim the production of composite particles in a variety of combinations including plastics, metals, ceramics (2-7). One important development in this area has been the use of the Mechano-fusion system developed by Hosokawa Inc. Senna et. al. have analyzed in detail the use of this system to synthesize composite particulates and analyze the ratio of the tangential stresses to the normal stresses for optimum conditions in the machine (1-2).

In this paper, we show the feasibility of a new method to form engineered particulates with controlled surface architecture. This method is based on the use of a magnetic field to accelerate magnetic particles which further collide with the core and the secondary particles. The magnetically assisted impaction coating (MAIC) process results in efficient mixing and mechanical deformation, thereby leading to the formation of adherent coatings. Particulate coatings consisting of different core (PMMA, alumina) and secondary particles (titania, alumina, silver, cobalt oxide, etc.) were used for the experiments. The MAIC coating method was compared with other standard blending techniques. Studies were conducted to understand the effect of the processing parameters such as particle size, and hardness on the surface coverage 


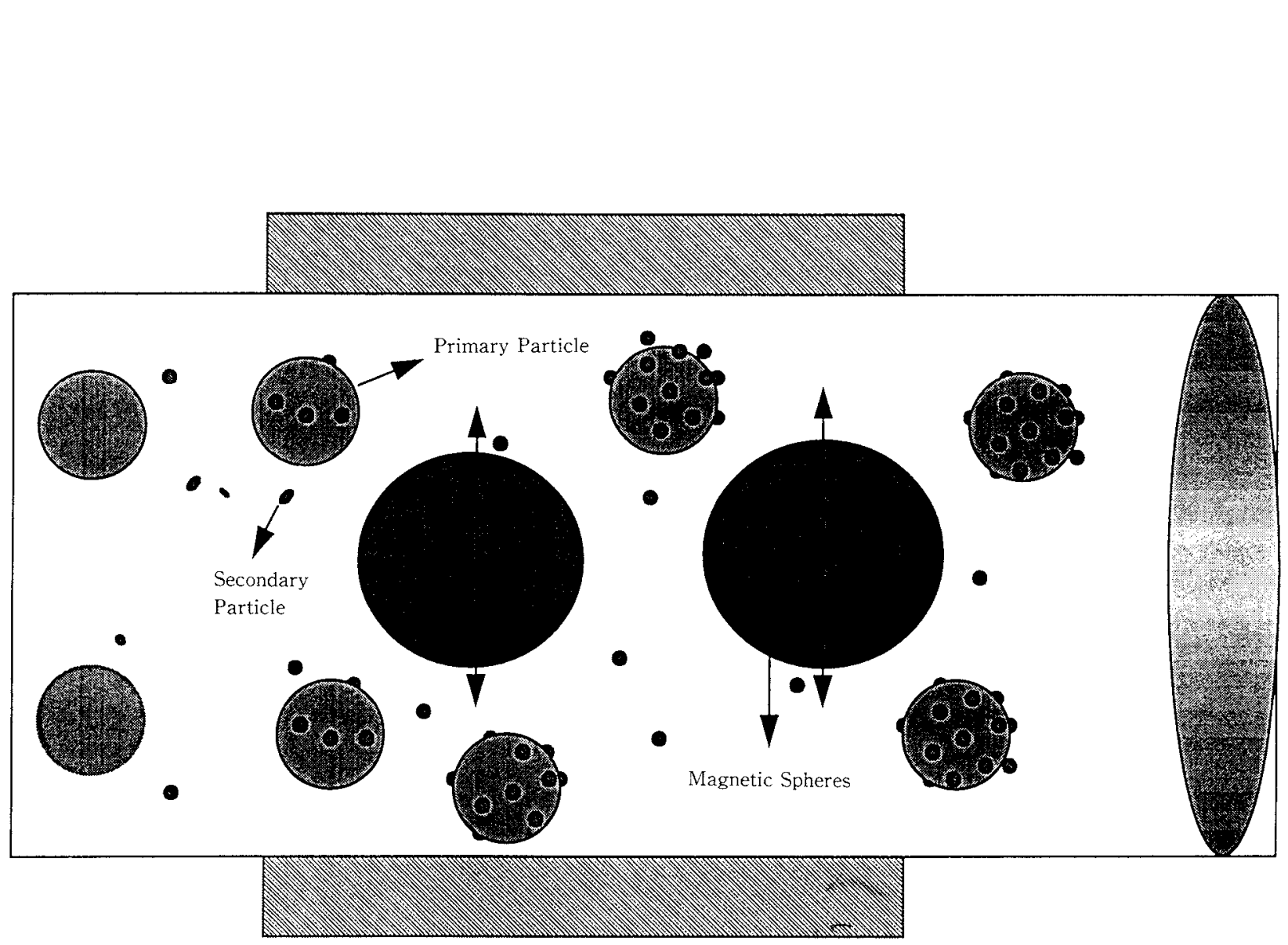

Fig. 2 Schematic diagram of the magnetically assisted impaction coating (MAIC) system

and degree of adhesion of the coating.

In order to understand the nature of adhesion in the composite particles, AFM studies were also conducted. Based on the experimental observations, the mechanisms for coating formation have been proposed.

\section{Experimental}

A schematic diagram of the magnetically assisted coating process (MAIC) is shown in Fig. 2. Briefly, this system consists of a long hollow cylindrical tube enclosed with a series of electromagnets. The flux inside the tube consists of the relatively large magnetic particles (size $200 \mu \mathrm{m}-1000 \mu \mathrm{m}$ ), core (size $1 \mu \mathrm{m}-200 \mu \mathrm{m}$ ) and secondary particles ( $5 \mathrm{~nm}-1$ $\mu \mathrm{m})$. Figure 3 shows the magnetization as a function of the applied magnetic field (B-H hysteresis loop) obtained from a SQUID magnetometer. An alternating current (with varying frequency and amplitude) is applied to the electromagnets, thereby creating a weak ( $\sim 100$ Gauss) magnetic field. The alternating magnetic field, produces by the time varying field which accelerates the magnetic particles within the system. The magnetic particles are characterized by a high coercivity (approx 4000 Gauss) so that they do not reverse their polarity in a weak electromagnetic field. Therefore, the mag. netic particles can be accelerated in the chamber. The magnetic particles in-turn collide with the core and secondary particles resulting in the formation of a psuedo-fluidized bed. As the magnetic response of each type of particle is different, the particle collide in a random manner. It is envisaged that these collisions lead to formation of a particulate coating.

After the formation of the coating, the magnetic particles are separated from the composite particles by standard magnetic separation methods. The adherence of the coating depends on a number of process parameters such as, electromagnetic field strength, frequency of the applied magnetic field, process time, particle loading and concentration etc. Materials parameters such as the hardness and

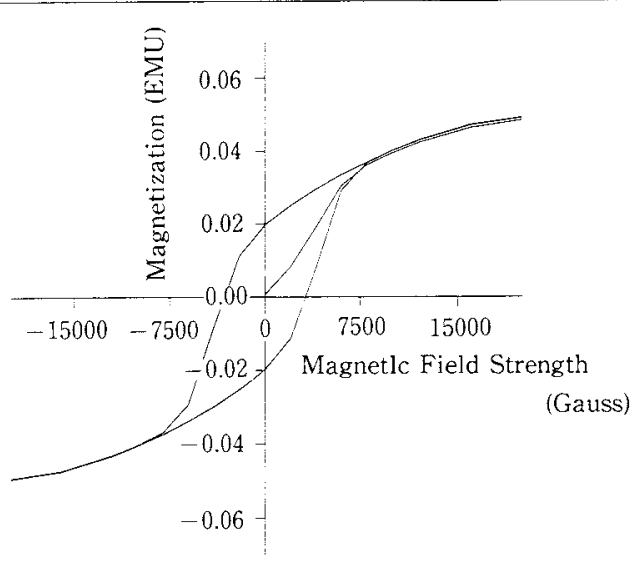

Fig. 3 Magnetization versus applied magnetic field for Iron Oxide used in the MAIC system 


\section{no $k$}

yield strength of the core and secondary particles, surface roughness and particle size also play a critical role in controlling the adhesion of the coating. In addition these parameters also control the surface architecture of the coating (distribution of particulates, particle density on the surface, etc.)

The composite engineered particulates were characterized for their surface architecture and composition, uniformity and texture by standard materials characterization techniques. Secondary electron microscopy (SEM) studies on these particles were conducted to determine the surface morphology of the coatings, while energy dispersive $\mathrm{X}$-ray analysis (EDX) and wavelength dispersive $\mathrm{X}$-ray analysis (WDX) were conducted to determine the surface composition and surface architecture of the films. It was observed that after the coating process, some of the particles appeared to be weakly adhering to the surface of the core particle. In some experiments, the composite particulates were treated to standard ultrasonic treatment (20 Khz, 1 minute) conditions, so that loosely attached/weakly adhered particles could be removed from the surface. The effect of deformation on adhesion characteristics was determined at the particulate level using atomic force microscopy (AFM) techniques and correlated with the particle ensemble behavior.

\section{Results}

\section{Comparison of Other Coating Techniques}

To determine the efficacy of the MAIC process, experiments were conducted and compared with standard blending techniques. These experiments were conducted with both soft-hard and hard-hard combination of secondary and core particles. Fig. 4(a) and Fig. 4(b) show the surface morphology at two different magnifications of fumed $\mathrm{SiO}_{2}$ coatings on silicon carbide core particles synthesized by the MAIC process. Both these particles are relatively hard (Vickers hardness exceeding $1000 \mathrm{Kg} / \mathrm{mm}^{2}$ ), thus significant plastic deformation is not expected to occur. The percentage of the weight ratio of fumed silica (average particle size $\sim 0.1 \mu \mathrm{m}$ ) was approximately 1\%. No ultrasonic treatment or other cleaning operations were conducted on these samples. The SEM micrographs show that the coating is distributed uniformly on the surface without formation of large agglomerates. From image anal$y$ sis, the surface coverage of the fumed silica was determined to be approximately $30 \%$. Fig. 5(a) and 5(b) show the surface morphology of ball-mill blend- (a)

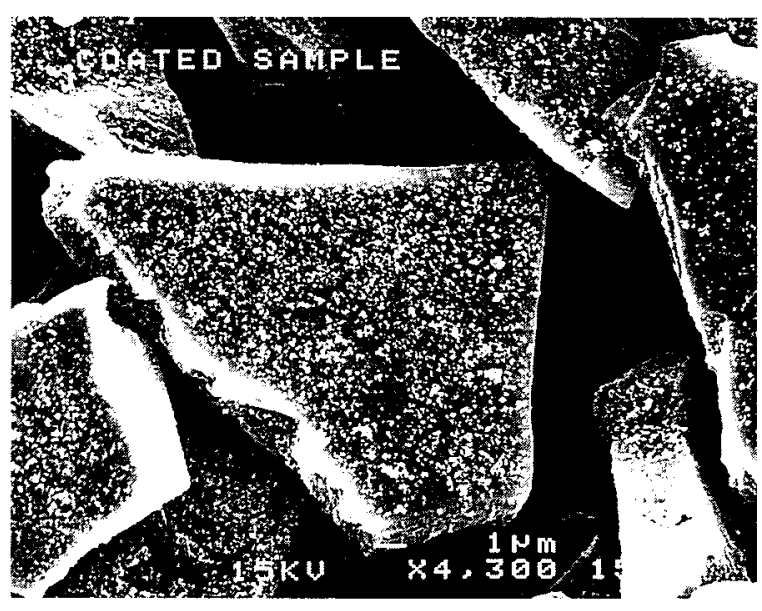

(b)

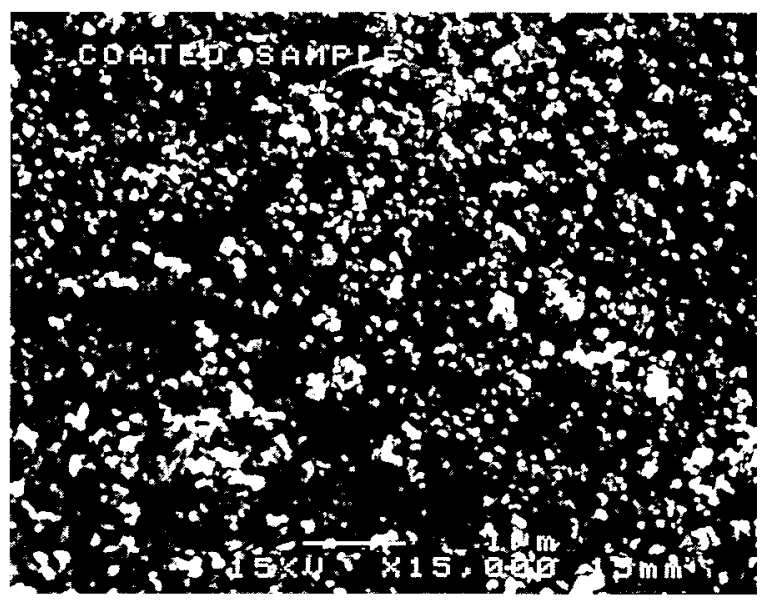

Fig. 4 SEM micrographs at (a) low and (b) high magnifica tion of fumed silica particles coated on silicon carbide particles by the MAIC process

ed $1 \%$ silica particles on silicon carbide core particles at two different magnifications. The micrographs show that the surface coverage of fumed silica particles on the silicon carbide surface was less than the MAIC treated samples by a factor of three, even though the same weight ratio of the materials was used. Thus, these results shows that the MAIC process is a highly efficient process in the formation of coatings. It should be noted that the adherence strength for either of the coatings could not be determined because no ultrasonication studies were carried out on these samples.

Similar results were obtained for soft-hard combination composite particulate combinations. Figure 6(a) and Figure 6(b) show the surface of a PMMA particles $(200 \mu \mathrm{m})$ coated with alumina with using (i) the MAIC process and (ii) standard blending. In these experiments the size of the secondary particles was approximately $0.2 \mu \mathrm{m}$. The weight ratio of the secondary to primary particles was adjusted so that under ideal conditions an 100\% surface coverage could be achieved. The figure shows that the 


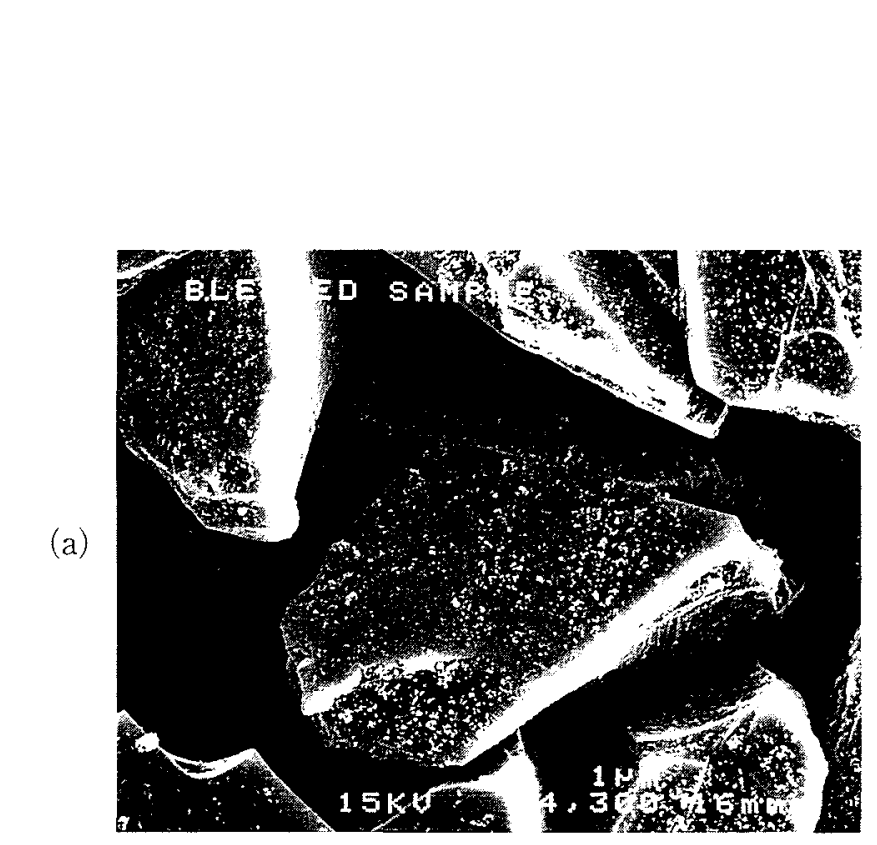

(a)

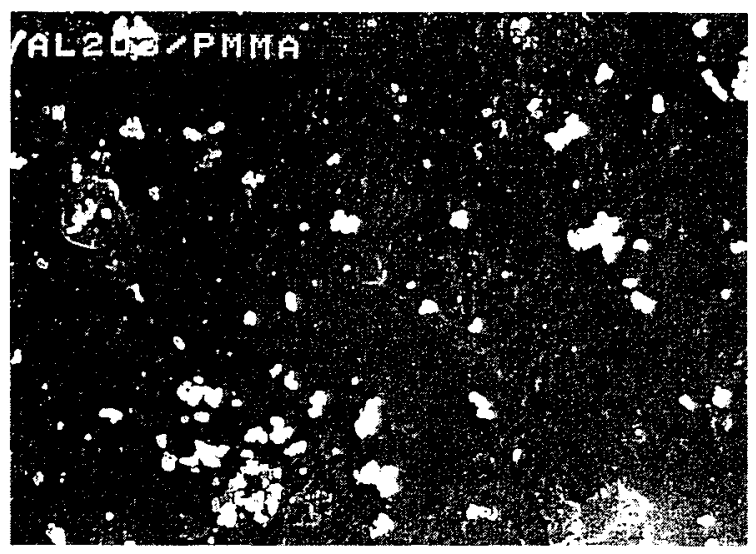

(b)

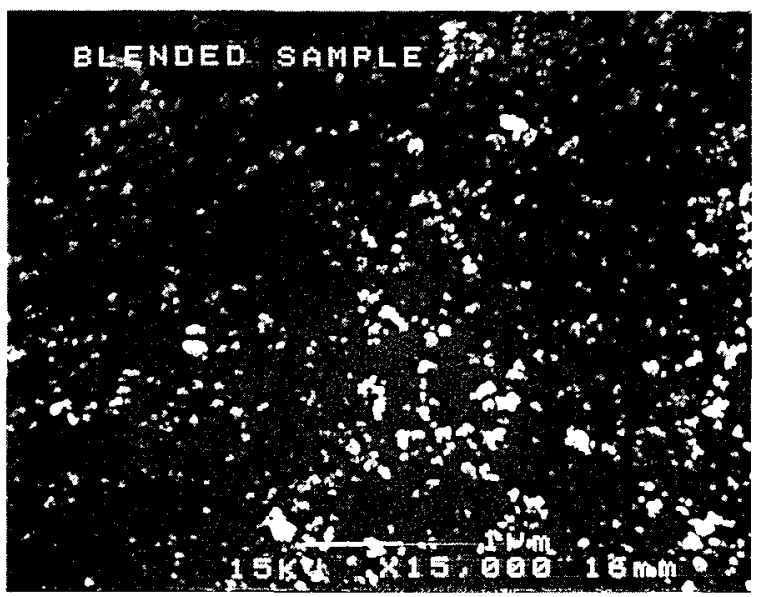

(b)

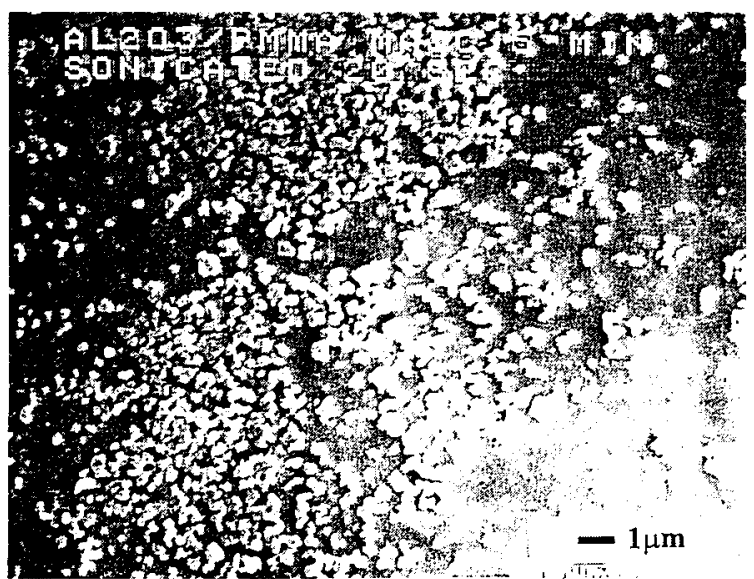

Fig. 5 SEM micrographs at (a) low and (b) high magnifica tion of fumed silica particles coated on silicon carbide particles by blending method

surface coverage of the coating made by the MAIC process was more than an order of magnitude higher than the surface coverage observed from the blending method. The surface coverage values after ultrasonic treatment for both blended and MAIC treated samples is listed in Table 1. The ultrasonic treatment was conducted so that the loosely held particles from the surface can be removed. No appreciable detachment of the secondary particles was observed after ultrasonication for MAIC treated samples. However significant detachment of the particles was observed for composite particles prepared by standard blending methods.

\section{Effect of Particle Size and Hardness}

The size of the secondary particles was found to be an important factor controlling the degree of surface coverage and the efficiency of the coating process. Alumina secondary particles with size varying from $0.05 \mu \mathrm{m}$ to $1 \mu \mathrm{m}$ and primary PMMA particles (size $\sim 200 \mu \mathrm{m}$ ) were used in these experiments. The weight ratio of the secondary to pri-

Fig. 6 Surface morphology of $0.4 \mu \mathrm{m}$ alumina particles coated on PMMA particles obtained from (a) MAIC system and (b) standard blending techniques

mary particles was adjusted so that under ideal conditions an 100\% surface coverage could be achieved. Figure 7 shows the surface coverage of

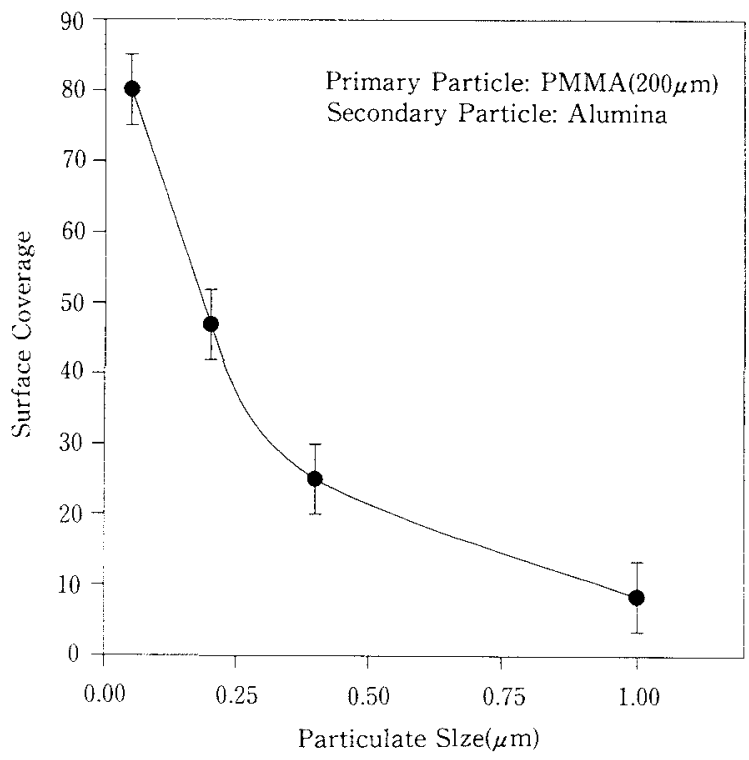

Fig. 7 Surface coverage of alumina particles on PMMA core particles as a function of particle size obtained from the MAIC method 
the alumina particles as a function of secondary particle size for $\mathrm{Al}_{2} \mathrm{O}_{3} /$ PMMA composite system. The surface coverage values were obtained from detailed image analysis of the SEM micrographs. No ultrasonic treatment or other cleaning techniques were applied on the particles. However it should be noted from Table 1, that the surface coverage of MAIC synthesized composite alumina/ PMMA particles do not decrease significantly upon ultrasonic treatment. The graph shows that the surface coverage is very high $(>80 \%)$ when the secondary particle size is less than $50 \mathrm{~nm}$, and drops to nearly $10 \%$ when the secondary particle size is increased to $1 \mu \mathrm{m}$. The maximum slope of the graph which corresponds to highest decrease in the coating coverage occurs when the secondary particle size varies from $0.1-0.3 \mu \mathrm{m}$ size range.

Table 1 Surface Coverage of the alumina particles on PMMA substrates before and after ultrasonication obtained from standard blending and MAIC techniques

\begin{tabular}{|c|c|c|c|}
\hline $\begin{array}{c}\text { Synthesis } \\
\text { Method }\end{array}$ & $\begin{array}{l}\text { Secondary } \\
\text { Particle Size } \\
(\mu \mathrm{m})\end{array}$ & $\begin{array}{l}\text { Surface } \\
\text { Coverage (\%) } \\
\text { (As synthesized) }\end{array}$ & $\begin{array}{l}\text { Surface } \\
\text { Coverage (\%) } \\
\text { (After } \\
\text { Uttrasonication) }\end{array}$ \\
\hline MAIC & 0.2 & $45 \pm 4$ & $40 \pm 4$ \\
\hline MAIC & 1 & $10 \pm 2$ & $6 \pm 2$ \\
\hline Blending & 0.2 & $18 \pm 3$ & $2 \pm 0.3$ \\
\hline Blending & 1 & $8 \pm 2$ & $0.6 \pm 0.1$ \\
\hline
\end{tabular}

The mechanical properties of the secondary and core particles play an important role in the adhesion of the coatings. Materials with lower hardness can deform upon impact, thus creating a larger impact area, and reduced roughness of the contacting surfaces. These effects lead to increased Van der Waals attraction forces. In addition, the secondary and core particles can approach closer to each other, thereby enhancing physical adhesion and increasing the possibility of chemical interactions between the two particles. Thus, it is expected that the adhesion strength of the secondary particle with the core particles will improve if at-least one of the particles possesses a low hardness.

Fig. 8(a) and Fig. 8(b) show respectively the surface morphology of alumina/ $\mathrm{TiO}_{2}$ particles before and after the ultrasonication process. In this experiment, the ratio of the secondary to primary particles was adjusted so that under ideal conditions, $100 \%$ surface coverage is obtained. The size of the primary and secondary particles were $10 \mu \mathrm{m}$ (a)

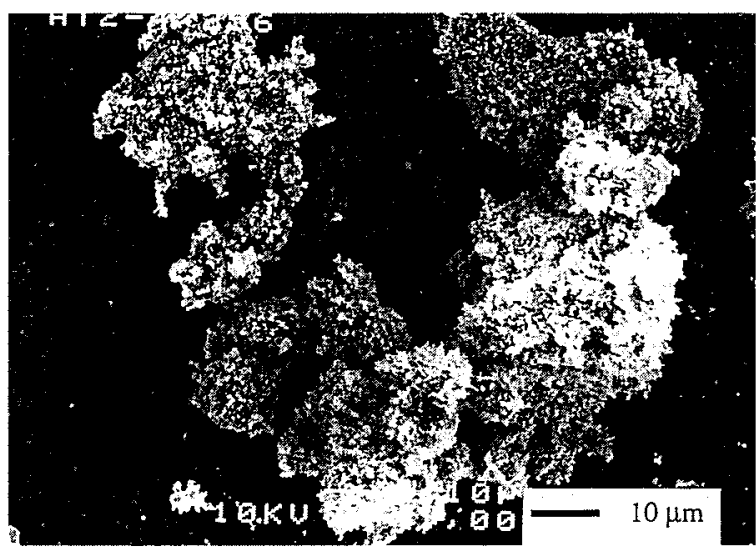

(b)

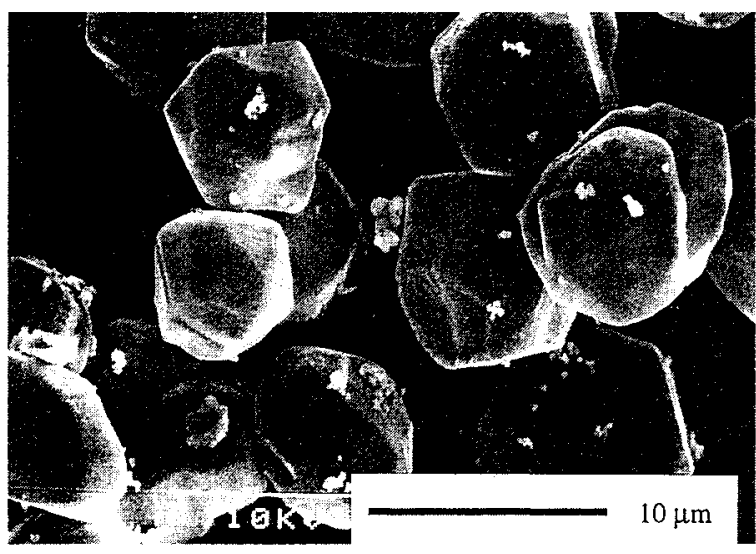

Fig. 8 Surface coverage of titania particles on alumina core particles after (a) MAIC synthesis and (b) MAIC synthesis followed by ultrasonication

and $0.4 \mu \mathrm{m}$, respectively. Also, as both particles are relatively hard, little plastic deformation effects are expected in this coating process. The figure shows that the synthesized titania coatings completely coat the primary particle. However after ultrasonication, a much lower surface coverage is observed. These results can be contrasted to the result obtained from soft-hard (alumina/PMMA) composite particles shown in Fig. 7, and Table 1. PMMA is relatively a softer material compared to alumina, thus deformation takes place on impact. In contrast, both titania and alumina are relatively hard materials and do not plastically deform under MAIC treatment conditions.

It should be noted that besides particle hardness, other factors area also crucial in controlling the surface architecture and the adhesion of coatings (8-9). For example, the surface roughness, and the chemical affinity of secondary and core particle surfaces is expected to play a critical role in the adhesion and surface coverage of the coatings. The effect of other system and particle variables on the surface coverage and the adhesion of the coatings will be discussed in detail in a future publication. 
ing surface. The deformation of the particle surface

\section{Discussion}

The above results shows that the intrinsic particle parameters such as hardness and particle size play a critical role in controlling the strength of adhesion and the surface coverage of the secondary particle coating. In this section, we outline the mechanisms which affect the formation of coating in the MAIC process. The primary forces which act to bring particles to the surface and hold it together are the Van-der Waals, electrostatic and the capillary forces (in presence of moisture) (9). As we are dealing with particle impact in the MAIC process, the present analysis will be limited to effect of particle impact in the Van der Waals attraction between the particles.

It is well known that non-polar and polar Van der Waals forces can be significantly increased if the cross-section area of contact between the particles can be increased. During particle impact, the deformation of the particle surface is expected to play a significant role in the adhesion process. The particles during impact may deform either by elastic or plastic deformation, however elastic deformation do not significantly increase the adhesion due to the stored energy effects (10). The amount of plastic deformation depends on the hardness of the secondary and the primary particle. As the secondary particle is much smaller than the core particle, the particle geometry can be approximated as a spherical particle attaching on a flat surface. The Van-der Waals adhesion force between the spherical particle and the flat surface is given by

$$
\mathrm{F}_{\mathrm{vdw}}=\frac{\mathrm{AD}}{12 z^{2}}
$$

where $z$ is the separation distance (approximated to be $4 \AA$ ) between the surfaces, $D$ is the particle diameter, $\mathrm{A}$ is the Hamaker's constant. In the above equation, the Van-der Waals forces have been assumed to come from ideal point contact with no deformation of the secondary or the core particle. As indicated earlier, depending on the intrinsic properties of the particles (primary and secondary), significant deformation of the particle can occur both as a result of the intrinsic adhesion forces as well as the externally applied impact forces. The additional Van der Waals force due to deformation is a function of the increased contact area caused by the deformation and is given by

$$
F_{\text {vdw(def) }}=\frac{A \rho^{2}}{6 z^{3}}
$$

where ' $\rho$ ' corresponds to the radius of the contact. can either be elastic or plastic in nature, however based on energy considerations, elastic deformations do not give rise to significant increase in the adhesion strength. The ratio of $F_{V_{d w}(d e f)}$ and $F_{V d w}$ represents and the adhesion force enhancement factor due to plastic deformation. The adhesion force enhancement factor is given by

$$
\frac{F_{\mathrm{VdW}(\text { def })}}{\mathrm{F}_{\mathrm{VdW}}}=\frac{2 \rho^{2}}{\mathrm{D} z}
$$

Figure 9 plots the adhesion force enhancement factor versus secondary particle diameter for two assumed deformation conditions (1\% and 10\% deformation). The percentage deformation is defined as the ratio of the plastic deformed radius to the particle radius expressed in a percentage value. This figure shows that there is a significant increase in the Van der Waals force due to plastic deformation. The adhesion force enhancement factor is higher for greater degree of deformation, and increases further when the diameter of the secondary particle is increased. This figure shows that impaction can lead to significant increase (factor of 10 to 100) increase in the Van-der Waals forces, which in turn can significantly increase the surface coverage and adhesion of the secondary particles. It should be noted the the amount of the deformation during the coating process will depend on the nature of impaction (particle velocity, size, angle) and the intrinsic properties of the particles (hardness, elastic properties, etc.) (10-12).

Several researchers have proposed models to calculate the contact radius between a particle and the substrate (13-16). A majority of the work has been concentrated on determination of the elastic contact radius. Derjaguin, Muller and Toporov

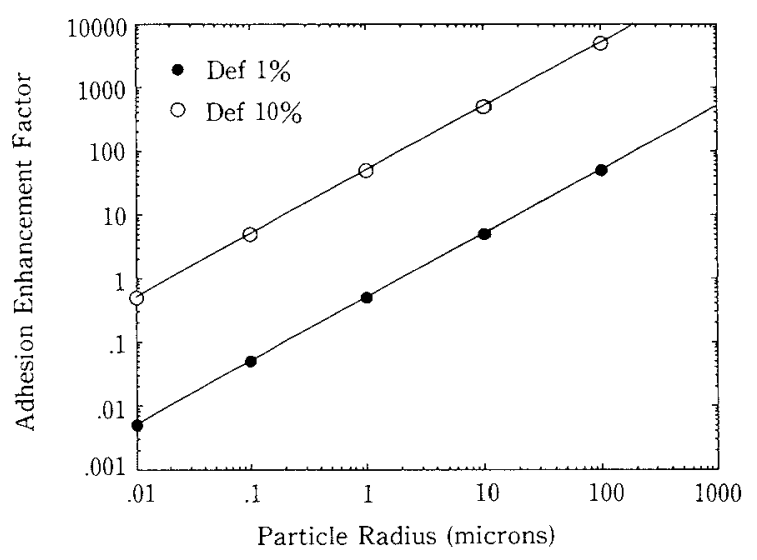

Fig. 9 The adhesion enhancement factor due to plastic parti. cle deformation versus size of the secondary particle. In this graph only the Van der Waals forces has been. shown. 
(hePeafter referred as the DMT model) proposed a model to calculate the contact radius by taking into account the tensile elastic interactions (14-15). Assuming that the shape of the contact region would be Hertzian, the DMT model predicts that half the interactions occurs outside the contact zone. This is in contrast to the Johnson, Kendall Roberts (JKR) model which assumes that all the interactions occur within the radius of contact (13). Further work in this area showed that both the models had a certain region of validity (16). However as mentioned earlier, the increase is contact radius with elastic deformation does not lead to an significant increase in the Van der Waals forces because of the "elastic rebound" effects.

Maguis and Pollock generalized the JKR model to include plastic deformation (17). They found out that in the case of plastic deformations, the contact radius " $\rho$ " is related to the particle radius $R$ and an externally applied load $\mathrm{P}$ by

$$
\mathrm{p}+2 \pi \mathrm{W}_{\mathrm{a}} \mathrm{R}=\pi \rho^{2} \mathrm{H}
$$

where $\mathrm{W}_{\mathrm{a}}$ is the thermodynamic work of adhesion, and the hardness " $\mathrm{H}$ " is related to the yield strength of the material $\mathrm{Y}$ by

$$
\mathrm{H}=3 \mathrm{Y}
$$

Equation 4 suggests that if the impact forces are much larger than the intrinsic adhesion forces, the radius of the deformed area will be directly proportional to the applied load and inversely proportional to the hardness of the contacting surfaces. Thus based on equation (4) and (5), we can conclude that the hardness of the particle, and the impact load will play a significant role in the adhesion process.

To understand the effect of the particle impact on the adhesion forces we conducted AFM indentation studies on various two substrates (PMMA and sapphire). PMMA is relatively a soft material (Vickers hardness $\sim 20 \mathrm{Kg} / \mathrm{mm}^{2}$ ), while sapphire corresponds to a hard surface (Vickers Hardness $\sim 2100 \mathrm{Kg} / \mathrm{mm}^{2}$ ). These studies were conducted by loading the substrate surface with silicon nitride AFM tips under different loading conditions to simulate the experimental system. Figure 10 shows the adhesion force on the AFM tip as a function of loading for two sapphire and PMMA substrates. This AFM experimental geometry simulates hardhard and hard-soft particle impaction in the MAIC system. These experiments were conducted in dry air conditions so that the capillary effects could be eliminated. The figure show that for PMMA substrates, the adhesion force increases linearly as the loading force is increased. The linear increase in the
Van der Waals forces is agreement with equations (2) and (4). In contrast, the adhesion forces on the sapphire substrates is independent of the loading force. This is attributed to the large hardness values of the substrate which does not result in plastic deformation.

The decrease in the surface coverage with increasing particle size for MAIC coated composite particle can be attributed to two effects (i) surface roughness of core and secondary particles and (ii) impaction effects. It is well known that the surface roughness plays a critical role in adhesion of materials (18). The van der Waals forces are significantly reduced due to asperities on the surface. Even though, theoretically the predicted Van der Waals forces for small particles $(<10 \mu \mathrm{m})$ are much greater than the gravitational forces, in reality the gravitational forces become higher because of the reduced Van der Waals attraction due to surface roughness.

Figure 11 shows the effect of surface roughness on the Van der Waals attraction as a function of diameter of the secondary particle. This plots are based on the Van der Waals attraction between two spheres of different size assuming smooth surface contact and rough surface contact (19). The rough surface can be simulated by attaching a hemispherical particle to a flat surface. The figure shows that when the size of the secondary particle is relatively large $(>1 \mu \mathrm{m})$, the Van der Waals forces for rough contacts are reduced by several orders of magnitude

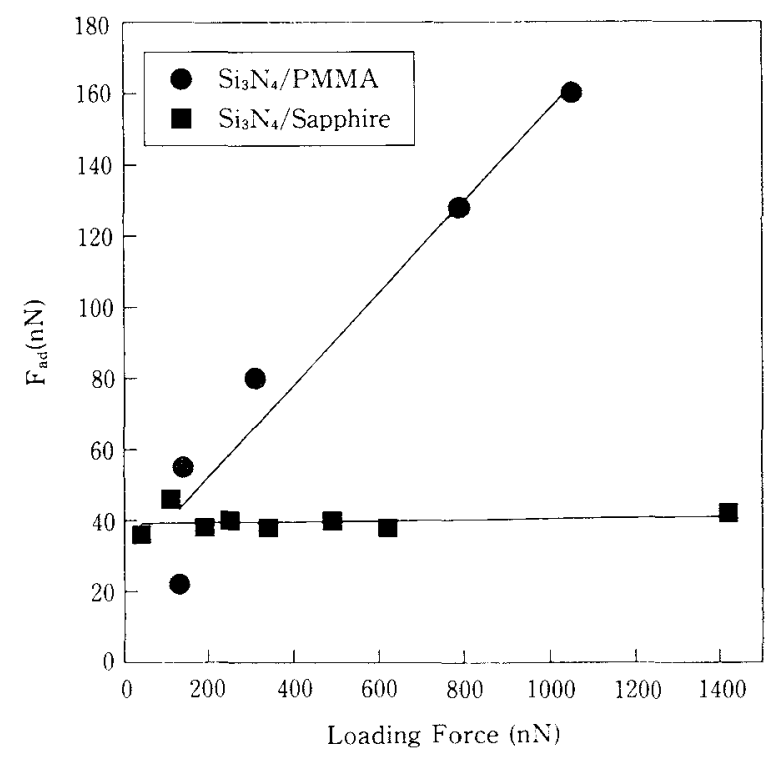

Fig. 10 The adhesion force between the AFM tip and PMMA and sapphire substrates as a function of loading. The loading leads to plastic deformation of the PMMA substrate while no plastic deformation in sapphire occurs. 


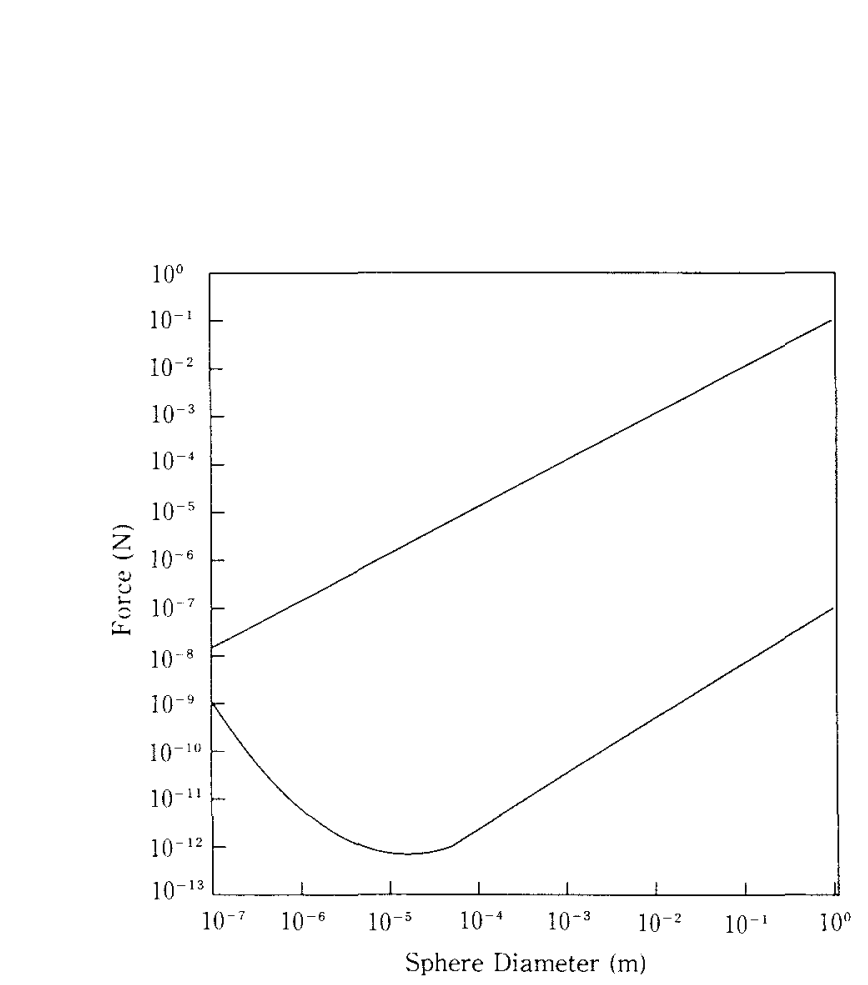

Fig. 11 Van der Waals forces between two spherical particles under (i) smooth contact and (ii) rough contact conditions

than expected from a smooth contact. However as the size of the secondary particle decreases, the Van der Waals for rough surfaces starts to increase. This increase is because the size of the asperity becomes comparable to the size of the secondary particle. As the size of the secondary particle is further decreased, the Van der Waals forces due to rough contact approaches the values expected from a smooth surface contact. Thus for small diameter secondary particles, Van der Waals forces becomes significantly larger. Similar effect of particle size has been obtained by the MAIC process (for example Alumina particles on PMMA). Significant secondary particle surface coverage has been obtained when the size of the secondary particle is small $(\sim 50 \mathrm{~nm})$. With increase in particle size the surface coverage decreases rather significantly. It should also be noted that the deformation of the surface during impaction is also expected to play a role in modification of the surface roughness of the primary and secondary particles.

The impaction stresses are also affected with the size of the secondary particle. The coating process takes place via direct collisions of various species (secondary particles, core particles and the magnetic particles). The process is envisaged to occur in two steps. This is shown schematically in Fig. 12. In this figure we have assumed a soft core particle and a hard secondary particle. The first step involves random collisions the secondary particles with primary particles resulting in weak attachment. In the second step, collisions with large magnetic particles results in deformation of the

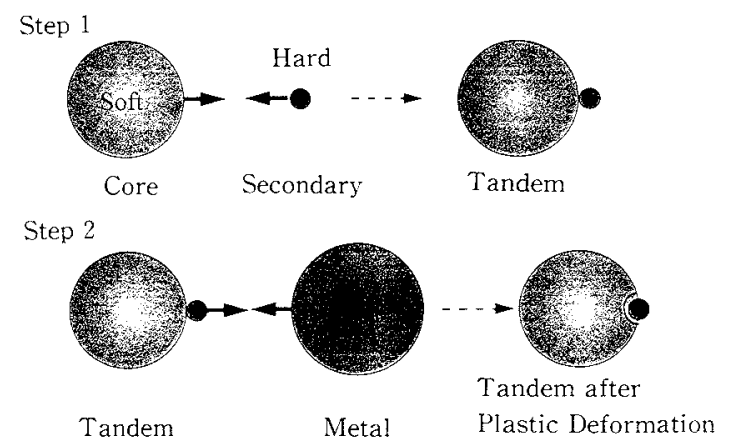

Fig. 12 Schematic diagram to deformation mechanisms which results in improved adhesion of the hard-soft coatings synthesized by the MAIC process

primary particle, thereby enhancing attractive forces. For a fixed external impact load, the surface stresses increase with decreasing secondary particle size. Thus, the smaller secondary particles will cause larger deformation to occur, which result in increased Van der Waals attraction.

\section{Conclusions}

A novel dry method for particulate coatings has been developed. This method is based on the use of a magnetically assisted impaction process to induce uniform mixing, deformation and improve adhesion strength of the coating. The MAIC process for coating particles was found to be much more efficient than standard ball-mill blending techniques. Results show that the coating characteristics (surface coverage, adhesion strength) is strongly affected by the materials parameters such as particle hardness and size. Based on the experimental observation models to explain the nature of the coatings process in the MAIC system has been developed.

\section{Acknowledgements}

Part of this research is sponsored by the National Science Foundation funded Engineering Research Center on Particle Science and Technology via grant \# EEC 94-0929. The authors would like to thank Brij Moudgil and Dinesh Shah for thought provoking discussions, and David Wright for helping to set-up the experiments.

\section{References}

1) K. Mizota, S. Fujiwara, and M. Senna, "Microstructure and Adhesion Mechanism of Mechanically Prepared Composite Particles" Materials Science and Engr. B 10, 
Ni39 (1991)

2) T. Yokoyama, K. Urayama, M. Naito, "The Angmill Mechanofusion System and its Applications" Kona, 559 (1987)

3) M. Satoh et. al., Proc 2nd World Congress on Particle Technology, 591 (1990)

4) M. Alonso, M. Satoh, K. Miyanami, "Mechanism of Combined Mechanofusion Processing of Powders" Powder. Tech. 59, 45 (1989)

5) M. Alonso, M. Satoh, K. Miyanami, "Powder Coating in a Rotary Mixer with rocking Motion" Powder Tech. 56, 135 (1988)

6) R.K. Singh "Dry Coating Method for surface modification of Particulates" Proceedings of the 10th International Conference on Surface Modification Technologies (in press)

7) R.K. Singh et. al., Appl. Phys. Lett, submitted for publication

8) D.S. Rimai, L.P. Demejo and R.C. Bowen, "Mechanics of Particle Adhesion" J. Adh. Sci \& Tech. 8, 1333 (1994)

9) R.A. Bowling ": Particles on Surfaces 1: Detection Adhesion and Removal" K.L. Mittal (eds.) Plenum Press NY (1989) pg. 129

10) J. Reed, "Particles on Surfaces $2:$ Detection Adhesion and Removal" Edited by K. Mittal, Plenum Press pg .1,
(1989)

11) S. Wall, W. John and S.L. Goren, "Particles on Surfaces 2: Detection Adhesion and Removal", Edited by K. Mittal, Plenum Press pg .19, (1989)

12) K. Kendall and J.C. Padget, "Latex Coalescence" Intl .J. Adhesion Adhesives, 2, 149 (1982)

13) K.L. Johnson, K. Kendall and A.D. Roberts, "Surface energy and Contact of elastic solids" Proc. R. Soc. London Ser. A 324, 301 (1971)

14) B.V. Derjaguin, V.M. Muller and Y.P Toporov, "Effect of Contact Deformations on the Adhesion of Particles" $\mathrm{J}$. Colloid and Interface Science 53, 314 (1975)

15) B.V. Derjaguin, V.M. Muller and Y.P Toporov, "On the Role of Molecular Forces in Contact Deformations" J. Colloid and Interface Science 67, 378 (1978)

16) D. Tabor, "Surface forces and surface Interactions" J. Colloid and Interface Science 58, 2 (1978)

17) D. Maugis and H.M. Pollock, "Surface Forces, Deforma tion and Adherence at Metal Microcontacts" Acta Metall, 32, 1323 (1984)

18) K. Kendall, Science, "Adhesion: Molecules and Mechanics" Vol 263, pg 1720 (1994)

19) H. Rumpf and H. Schubert, in "Ceramic Processing Before Firing", pg 357 edited by G.Y. Onoda and Larry L. Hench, J. Wuley and Sons, 1978 


\section{Author's short biography}

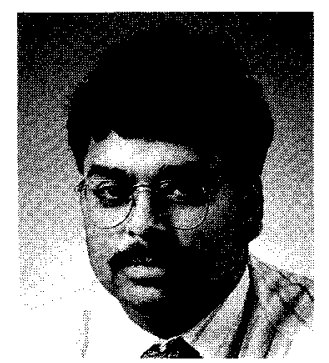

\section{Rajiv. k. Sinsh}

Dr. Rajiv Singh is currently a professor in the Materials Science \& Engineering Department at the University of Florida. He has published over 200 papers in the area of innovative synthesis and processing of materials, thin film coatings, surface modification and photon solid interaction based processing of materials. Prof. Singh received his $\mathrm{Ph}$. D degree from North Carolina State University in 1989. He is the reception of several awards involving NSF Young Investigation (NY1) award and TMS Hardy Gold Medal for outstanding contributions in materials science and engineering.

\section{Ali Ata}

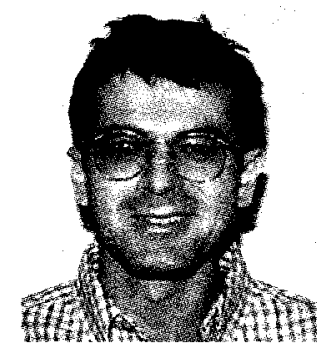

Ali Ata graduated in Metallurgical Engineering from Middle East Technical University of Ankara Turkey and obtained his Masters degree in Materials Science from Technical University of Istanbul in 1993. He has done two years of research at the University of California, San-Diego and subsequently started his $\mathrm{PhD}$ program in Materials Engineering at the University of Florida. He is currently in the program studying Engineering Particulate Systems, adhesion phenomena in particulate system.

\section{James Fitz-Gerald}

James Fitz-Gerald is a graduate student in Materials Engineering at the University of Florida. He obtained his B.S degree in Mechanical Engineering from University of Massachuset, Lowell. His current research is on the development of thin coatings on particulate materials.

\section{Ya. I. Rabinovich}

Dr. Yakov Rabinovich is a scientist in Engineering Research Center at the University of Florida. His major research interest is surface forces between particles in gas and liquid media including adhesion of micro-particles.

\section{W. Hendrickson}

Dr. Hendrickson is the president of Aveka Inc. at Minnesota. He got his Ph.D in Chemical Engineering from the University of Florida and worked in $3 \mathrm{M}$ company. He is working on particulate processing including dry coating of particulate materials. 